\title{
BMJ open Effect of specific resistance training on forearm pain and work disability in industrial technicians: cluster randomised controlled trial
}

\author{
Lars L Andersen, ${ }^{1}$ Markus D Jakobsen, ${ }^{1}$ Mogens T Pedersen, ${ }^{2}$ \\ Ole S Mortensen, ${ }^{3}$ Gisela Sjøgaard, ${ }^{4}$ Mette K Zebis ${ }^{1,4}$
}

To cite: Andersen LL, Jakobsen MD, Pedersen MT, et al. Effect of specific resistance training on forearm pain and work disability in industrial technicians: cluster randomised controlled trial. BMJ Open 2012;2:e000412. doi:10.1136/

bmjopen-2011-000412

- Prepublication history for this paper is available online. To view these files please visit the journal online (http:// dx.doi.org/10.1136/ bmjopen-2011-000412).

Received 23 September 2011 Accepted 25 January 2012

This final article is available for use under the terms of the Creative Commons Attribution Non-Commercial 2.0 Licence; see http://bmjopen.bmj.com

${ }^{1}$ National Research Centre for the Working Environment, Copenhagen, Denmark

${ }^{2}$ Department of Exercise and Sport Sciences, University of Copenhagen, Copenhagen, Denmark

${ }^{3}$ Department of Occupational Health, Bispebjerg University Hospital, Copenhagen, Denmark

${ }^{4}$ Institute of Sports Science and Clinical Biomechanics, University of Southern Denmark, Odense, Denmark

\section{ABSTRACT}

Objectives: To determine the effect of specific resistance training on forearm pain and work disability in industrial technicians.

Design and setting: Two-armed cluster randomised controlled trial of 20 weeks performed at two industrial production units in Copenhagen, Denmark.

Participants: Working-age industrial technicians both with and without pain and disability.

Interventions: The training group $(n=282)$ performed specific resistance training for the shoulder, neck and arm muscles three times a week. The control group $(n=255)$ was advised to continue normal physical activity.

Outcome: All participants rated forearm pain intensity (Visual Analogue Scale, 0-100 mm) once a week (primary outcome) and replied to a questionnaire on work disability (Disability of the Arm Shoulder and Hand, $0-100$ ) at baseline and follow-up (secondary outcome).

Results: Questionnaires were sent to 854 workers of which $30(n=282)$ and $27(n=255)$ clusters were randomised to training and control, respectively. Of these, 211 and 237 participants, respectively, responded to the follow-up questionnaire. Intention-totreat analyses including both individuals with and without pain showed that from baseline to follow-up, pain intensity and work disability decreased more in the training group than in the control group (4-5 on a scale of $0-100, p<0.01-0.001)$. Among those with pain $>30 \mathrm{~mm}$ Visual Analogue Scale at baseline $(n=54)$, the OR for complete recovery at follow-up in the training group compared with the control group was $4.6(95 \% \mathrm{Cl} 1.2$ to 17.9$)$. Among those with work disability $>30$ at baseline $(n=113)$, the $O R$ for complete recovery at follow-up in the training group compared with the control group was $6.0(95 \% \mathrm{Cl} 1.8$ to 19.8 .

Conclusion: Specific resistance training of the shoulder, neck and arm reduces forearm pain and work disability among industrial technicians.

Trial registration number: NCT01071980.

\section{ARTICLE SUMMARY}

Article focus

- Forearm pain is associated with work disability and healthcare costs.

- Specific resistance training effectively reduces neck and shoulder pain, but its effect on forearm pain is only scarcely investigated.

- Our study determines the effect of specific resistance training on forearm pain and work disability in industrial technicians.

Key message

- Specific resistance training of the shoulder, neck and arm reduces forearm pain and work disability among industrial technicians.

Strengths and limitations of this study

- The cluster randomised controlled design with high statistical power strengthens our study.

- As we included both public and private sector companies of which most of the invited employees agreed to participate, the external validity of our findings is high.

- The inclusion and exclusion criteria limit the generalisability to technicians with non-specific forearm pain.

- A limitation is the loss to follow-up of more than $20 \%$ of the participants in the training group.

\section{INTRODUCTION}

Musculoskeletal disorders are associated with considerable work disability, healthcare costs and sickness absence. ${ }^{1-3}$ Almost all employees depend on well-functioning upper extremities for daily work tasks. Frequent repetitive movements of the arm or wrist have been associated with development of forearm pain ${ }^{4}$ and lateral epicondylitis (tennis elbow). ${ }^{5} \mathrm{~A}$ recent systematic review quantified the dose-response relationship between work exposure and disorders and reported that repetitive movements for more 
than $2 \mathrm{~h}$ a day increased the risk for lateral epicondylitis. ${ }^{6}$ Data from 2003 showed that the 1-year incidence of forearm pain among newly employed workers across different occupations was $8 \%{ }^{4}$ and the weekly prevalence of moderate-to-severe forearm pain among computer users was $4 \% .^{7}$ The aetiology of forearm pain is related to both physical and psychosocial distress. ${ }^{8} 9$ As many occupations inherently involve hand-arm exposure complementing interventions to support existing workplace, ergonomic adjustments should be considered.

General health benefits of regular physical exercise are well known. ${ }^{10}$ As most adults spend many hours at work several days a week, the workplace provides a feasible setting for health promotion. While previous studies have documented the effectiveness of workplace interventions with physical exercise for preventing and relieving neck/shoulder and back pain, ${ }^{11}$ none of these studies concerned forearm pain. Randomised controlled trials have shown mixed results on the effectiveness of specific resistance training for specific forearm disorders in patients, for example, lateral epicondylitis. ${ }^{12}{ }^{13} \mathrm{We}$ have shown in one study that upper body resistance training can to some extent reduce pain symptoms of the elbow and wrist among office workers. ${ }^{14}$ However, the effect of such interventions on work disability is unknown. Thus, randomised controlled trials investigating the effect of workplace physical exercise interventions on forearm pain and work disability are needed. In workplace interventions involving the majority of employees, randomisation at the cluster level (eg, department) is preferred to avoid contamination of interventions. ${ }^{15}$

This cluster randomised controlled trial determines the effect of specific resistance training on forearm pain and work disability in industrial technicians. We hypothesise that specific resistance training reduces pain and work disability.

\section{METHODS}

\section{Study design}

We performed a cluster randomised controlled trial in Copenhagen, Denmark, from January (recruitment) to June 2009 (follow-up). All employed technicians from two large industrial production units from the public and private sector were invited to participate. The private sector company specialised in creating bioindustrial products by using enzymes and the public sector company specialised in production of vaccines and control of infectious diseases. At both companies, the work involved repetitive tasks, such as pipetting, preparing vial samples for analysis and data processing on a computer including mouse work, all tasks that require precision in work and may result in extended periods of time spent in static working postures.

Pain and disability were not specific inclusion criteria. The rationale for this was that musculoskeletal pain typically is episodic. Including all employees allowed us to study the effect of the intervention at the company level, as well as the treatment and preventive effect, respectively, among subgroups with and without pain at baseline. The main outcome on neck/shoulder pain is described elsewhere. ${ }^{16}$ Figure 1 shows the flow of participants through the trial.

In brief, we sent an internet-based questionnaire to 854 prospective participants of which 669 replied. One hundred and four declined to participate or did not reply to the question concerning participation. Exclusion criteria-which led to exclusion of 28 participants-were pregnancy and serious health conditions, such as previous trauma or injuries, life-threatening diseases and cardiovascular disease. Thereby 537 participants were included in the study and randomly assigned at the cluster level to training ( $\mathrm{n}=282$ in 30 clusters) or control ( $\mathrm{n}=255$ in 27 clusters) (table 1 ).

All participants gave their written informed consent to participate in this study, which conformed to the Declaration of Helsinki and was approved by the local ethical committee (HC2008103). Trial registration: http://ClinicalTrials.gov (NCT01071980).

\section{The cluster randomisation procedure}

Eligible volunteers were randomised at the cluster level to training or control. The statistician performing the cluster randomisation had no access to the baseline database. Departments were stratified into 14 strata according to the following nested criteria: company, type of work task and size of department. Strata were labelled alphabetically, and clusters were numbered consecutively within strata. This resulted in a total of 57 clusters with sizes ranging from 1 to 40 participants. A statistician blinded to the identity of the strata, and clusters assigned the clusters within each stratum by simple random allocation to either the training or the control. The consecutive numbers of the clusters within each stratum were written on pieces of paper and drawn from an opaque, tossed plastic bag. To minimise imbalance over several strata with odd numbers of clusters, these strata were paired, and clusters were alternately allocated to either training or control, the first cluster being allocated to either training or control depending on the flip of a coin. Thus, all clusters had the same chance of being allocated to the training group.

\section{Intervention}

The training group performed specific resistance training for the shoulder, neck and arm with dumbbells (wrist extension, shoulder lateral raise, shoulder front raise, shoulder shrugs, reverse flies) three times of $20 \mathrm{~min} /$ week for 20 weeks. Training loads were progressively increased from moderate loadings of 15-20 repetitions maximum (RM-ie, the number of repetitions that could be performed to momentary fatigue) during the initial weeks to relatively heavier loadings of $8-12$ (RM) during the final weeks. The exercises were performed in a slowly controlled manner avoiding sudden jerk and acceleration. Experienced instructors introduced the training programme in 


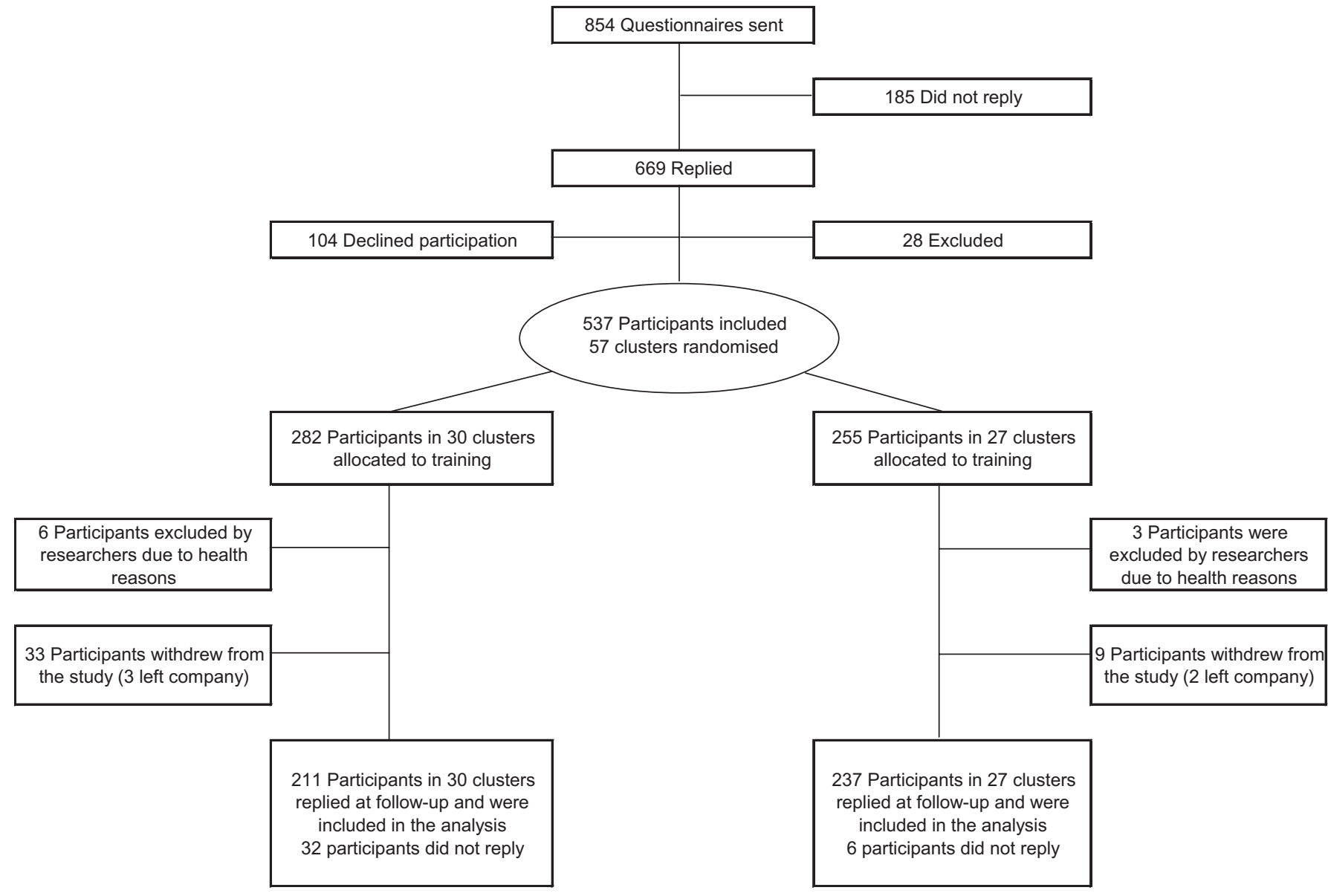

Figure 1 Flow of participants through the trial.

groups of 5-15 colleagues. After the introductory session, the participants had the opportunity to train individually or in self-organised groups. The instructors supervised every other training session throughout the 20 weeks to ensure correct technique and sufficient progression of the training. The supervised sessions were scheduled with assistance from the respective departments to best fit into daily working routines. The remaining training sessions were openly planned, meaning that the participants could train whenever it matched their daily work schedule. The training facilities were placed as near as possible to the workstations. In practice, training locations were established in corridors, storerooms and conference rooms (11 locations in total). All locations were equipped with a training poster, a clock, chairs, two pairs of lifting straps and dumbbells (pairs of $1-25 \mathrm{~kg}$ ). Participants of the control group were advised to continue their normal physical activity as usual.

\section{Adherence}

Participants of the training group logged their training in a diary during each training session. Adherence was defined as the total number of training sessions as a percentage of the maximal possible number of training sessions during the 20 weeks (ie, 60 sessions).

\section{Forearm pain and work disability}

The outcomes were based on questionnaire replies. Therefore, blinding of 'outcome assessors' does not apply for the present study. In principle, the participants were outcome assessors as we used subjective rating scales. Participants could not be blinded, that is, after the randomisation, each participant was informed about his/her respective group allocation. However, the statistical analyses remained blinded by assigning each group an arbitrary number until after the pre-planned analyses were run.

\section{Primary outcome}

Once a week, participants of both groups rated forearm pain intensity at its worst during the previous 7 days on a $100 \mathrm{~mm}$ Visual Analogue Scale (VAS), where $0 \mathrm{~mm}$ is 'no pain at all' and $100 \mathrm{~mm}$ is 'worst possible pain'. ${ }^{17}$

\section{Secondary outcome}

Participants rated work disability at baseline and followup by the work module of the Disability of the Arm Shoulder and Hand (DASH) questionnaire, which has previously been validated among industrial workers ${ }^{18}$ : "In the past week did you have any difficulty:" (1) "using your usual technique for your work?", (2) "doing your usual work because of arm, shoulder or hand pain?", 
Table 1 Demographics, clinical and work-related characteristics of the participants at baseline

\begin{tabular}{|c|c|c|c|c|c|c|}
\hline & \multicolumn{2}{|c|}{ Randomised } & \multicolumn{2}{|c|}{ Pain cases } & \multicolumn{2}{|c|}{ Disability cases } \\
\hline & Control & Training & Control & Training & Control & Training \\
\hline Number of participants & 255 & 282 & 22 & 32 & 44 & 69 \\
\hline \multicolumn{7}{|l|}{ Demographics } \\
\hline Age, year & $43(9)$ & $44(11)$ & $45(9)$ & $45(11)$ & $43(9)$ & $45(10)$ \\
\hline Height, cm & $170(8)$ & $168(7)$ & $171(8)$ & $166(7)$ & $170(6)$ & $168(7)$ \\
\hline Weight, kg & $73(14)$ & $70(14)$ & $72(12)$ & $69(17)$ & $71(14)$ & $69(14)$ \\
\hline Body mass index, $\mathrm{kg} / \mathrm{m}^{2}$ & $25(5)$ & $25(4)$ & $25(4)$ & $25(5)$ & $25(5)$ & $24(4)$ \\
\hline Women (\%) & $80 \%$ & $90 \%$ & $86 \%$ & $97 \%$ & $93 \%$ & $90 \%$ \\
\hline \multicolumn{7}{|l|}{ Clinical } \\
\hline Forearm pain intensity (0-100 mm VAS) & $10(16)$ & $12(18)$ & $52(19)$ & $52(17)$ & $23(28)$ & $18(24)$ \\
\hline Work disability $(0-100)$ & $15(21)$ & $19(22)$ & $36(26)$ & $26(24)$ & 54 (19) & $50(18)$ \\
\hline \multicolumn{7}{|l|}{$\begin{array}{l}\text { Percentage of participants spending more } \\
\text { than half of total work time }\end{array}$} \\
\hline Working with the hands twisted or flexed & $27 \%$ & $34 \%$ & $45 \%$ & $44 \%$ & $36 \%$ & $41 \%$ \\
\hline $\begin{array}{l}\text { Doing the same finger movements several } \\
\text { times a minute }\end{array}$ & $58 \%$ & $65 \%$ & $73 \%$ & $56 \%$ & $75 \%$ & $80 \%$ \\
\hline $\begin{array}{l}\text { Doing the same arm movements several } \\
\text { times a minute }\end{array}$ & $35 \%$ & $39 \%$ & $50 \%$ & $44 \%$ & $52 \%$ & $54 \%$ \\
\hline Working in a static posture & $48 \%$ & $51 \%$ & $64 \%$ & $44 \%$ & $73 \%$ & $68 \%$ \\
\hline \multicolumn{7}{|l|}{ Other work-related characteristics } \\
\hline Weekly working hours & $35(8)$ & $35(8)$ & $36(8)$ & $35(9)$ & $36(5)$ & $34(9)$ \\
\hline Years working as technician & $15(11)$ & $16(12)$ & $18(11)$ & $21(14)$ & $16(11)$ & $18(13)$ \\
\hline
\end{tabular}

(3) “doing your work as well as you would like?" and (4) "spending your usual amount of time doing your work?". Participants replied on a 5-point Likert scale from "no difficulty' to 'unable'. For comparability with VAS pain scores, the work disability score was normalised on a scale of $0-100$, where 100 represents the highest level of disability. ${ }^{19}$

Kaergaard and coworkers $^{20}$ found that for four complaint questions using 10-point scales, a sum of 12 (ie, average 3 for each of the four questions) was significantly associated with clinical findings. Because we used scales from $0-100$, we defined pain and disability cases accordingly as those scoring more than 30 on a scale of $0-100$.

\section{Sample size}

A priori power analyses showed that a sample size of 120 participants in each group would provide a power of $80 \%$ to detect a $15 \%$ change in pain. At an estimated dropout or loss to follow-up of $20 \%$, the minimally required number of participants in each group should be 150 in a design with simple randomisation. Calculations based on neck pain intensity from a previous cluster randomised study ${ }^{21}$ showed that the intra-cluster correlation $(\rho)$ was close to zero. However, we made a conservative estimate and assumed that $\rho$ would be equal to 0.1 and the average cluster size $(\mathrm{m})$ would be equal to 10 . Using the formula $1+(\mathrm{m}-1) \rho,{ }^{15}$ we would then need 1.9 times the sample size compared with not having used simple randomisation, that is, 285 participants in each group.

\section{Statistics}

We performed all analyses in accordance with the intention-to-treat principle and used a generalised linear mixed model (GLIMMIX procedure of SAS) controlled for gender to determine differences in the main outcomes between the two groups from baseline to follow-up. We added a random cluster effect to the model to account for possible intra-cluster correlations. We did not impute missing data as all methods of data imputation have limitations. ${ }^{22}$ The GLIMMIX procedure inherently accounts for missing data.

Work disability was registered twice, at baseline and follow-up. Pain intensity was registered weekly, and therefore, each participant could have up to 20 registrations. To more accurately report the effect of the intervention on pain, we used linear regression for each participant to estimate the trend over time and thereby determined the change from baseline to follow-up by multiplying the slope with the number of weeks. For the linear regression on pain intensity, we included participants with at least two observations at least 10 weeks apart. The individual changes from baseline to follow-up were then entered in the GLIMMIX procedure. These analyses were pre-planned.

\section{Treatment and prevention of symptoms}

Using logistic regression analysis controlled for gender and age (Logistic procedure of SAS), we also calculated ORs for complete recovery at follow-up (defined as pain intensity or disability score of $<10$ ) among cases at baseline (defined as pain intensity or disability score of 
30 or more). Similarly, we calculated OR for becoming a case (score of 30 or more) at follow-up among those without symptoms (score of $<10$ ) at baseline. These analyses were exploratory.

We used the SAS statistical software V.9.2 for the analyses (SAS institute) and accepted an $\alpha$ level of $5 \%$ as statistically significant. We report baseline results as means (SD) and changes from baseline to follow-up as means $(95 \%$ CIs) unless otherwise stated.

\section{RESULTS}

At baseline, there were no significant differences between the two groups regarding demographics, clinical and work-related characteristics (table 1). For the two groups combined, forearm pain and work disability were moderately related (Spearman's $r=0.41$, $\mathrm{p}<0.0001)$.

During the 20-week period, participants from the training and control groups, respectively, had 12 (SD: 6) and 14 (SD: 5) weekly pain registrations. Based on the training diary registrations, participants from the training group followed the programme on average 36 times during the 20-week period, corresponding to an average adherence of $60 \%$. Training load of the specific forearm exercise-wrist extension-was approximately doubled during the training period and increased from 2.3 (1.1) $\mathrm{kg}$ during the initial 4 weeks to 4.3 (1.4) $\mathrm{kg}$ during the final 4 weeks. Similar improvements were observed for the shoulder exercises.

\section{Intention-to-treat effect}

Figure 2 shows the change in forearm pain from baseline to follow-up among the total study population. A priori hypothesis testing of main effects showed a significant group by time effect for forearm pain intensity $(\mathrm{p}<0.001)$ and work disability $(p<0.01)$. Intention-to-treat analyses showed that from baseline to follow-up, pain intensity decreased significantly more in the training group than in the control group, with a between-group difference of $5.2 \mathrm{~mm}$ VAS (95\% CI 2.3 to $8.1 \mathrm{~mm}$ VAS). Likewise, work disability decreased significantly more in the training group, with a between-group difference of 4.4 (95\% CI 1.4 to 7.5$)$.

Treatment effect among employees with pain and disability Among employees with pain $>30 \mathrm{~mm}$ VAS at baseline $(n=54), 69 \%$ and $31 \%$ from the training and control groups, respectively, completely recovered (VAS score of $<10 \mathrm{~mm})$. Pain decreased in the training group $(-21.1 \mathrm{~mm}, 95 \% \mathrm{CI}-32$ to -11$)$ but not in the control group ( $-4.5 \mathrm{~mm}, 95 \% \mathrm{CI}-16$ to 7$)$. The between-group difference in pain from baseline to follow-up was significant $(16.7 \mathrm{~mm}, 95 \%$ CI 0.8 to 32.5$)$. The OR for complete recovery at follow-up in the training group compared with the control group was 4.6 (95\% CI 1.2 to 17.9).

Among employees with work disability (DASH) $>30$ at baseline $(n=113), 77 \%$ and $36 \%$ from the training and

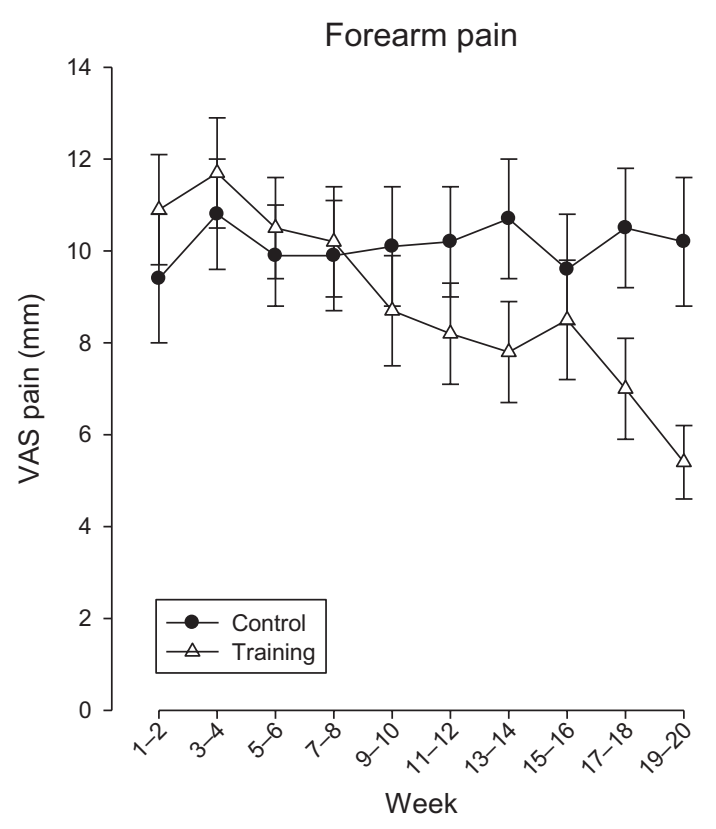

Figure 2 Illustration of the time-wise change in forearm pain for the training and control groups. The between-group difference from baseline to follow-up was of $5.2 \mathrm{~mm}$ Visual Analogue Scale (VAS) for training versus control $(95 \% \mathrm{Cl} 2.3$ to 8.1 ). Notice that the scale ranges from $0-100$.

control groups, respectively, recovered completely (disability score of $<10$ ). Work disability decreased both in the training group $(-36,95 \%$ CI -43 to -29$)$ and in the control group $(-26,95 \%$ CI -33 to -18$)$. The between-group difference in work disability from baseline to follow-up was significant (11,95\% CI 2.5 to 19), that is, the training group improved significantly more than the control group. The OR for complete recovery at follow-up in the training group compared with the control group was 6.0 (95\% CI 1.8 to 19.8).

\section{Preventive effect among symptom-free employees}

Among those without pain at baseline $(<10 \mathrm{~mm}$ VAS, $\mathrm{n}=286$ ), there was a small but statistically significant increase in pain in the control group $(1.6 \mathrm{~mm}, 95 \% \mathrm{CI}$ 0.5 to 2.7$)$ and not in the training group $(0.4 \mathrm{~mm}, 95 \%$ CI -0.7 to 1.6$)$. This change was not significantly different between the groups $(1.1 \mathrm{~mm}, 95 \% \mathrm{CI}-0.5$ to 2.8).

Among those without work disability at baseline (DASH $<10, n=271$ ), there was a small but statistically significant increase in work disability in the control group (2.2, 95\% CI 0.7 to 3.6 ) and not in the training group (1.8, $95 \% \mathrm{CI}-0.01$ to 3.5$)$. This change was not significantly different between the groups $(0.4,95 \%$ CI -1.6 to 2.4$)$.

\section{Adverse events}

Adverse events were minor and transient. Fifteen participants of the training group contacted the physical therapist of the project because of worsening of pain during training and ascribed these symptoms to previous 
injuries being provoked during training. With adjustment of the training programme-for example, reducing intensity or range of motion-these participants were able to complete the 20-week programme. Another four participants in the training group withdrew from the study due to worsening of pain during training. These participants did not consult the physical therapist prior to withdrawal.

\section{DISCUSSION}

Our study documents positive effects of specific upper limb resistance training on forearm pain and work disability in industrial technicians.

The baseline data of our study show that many industrial technicians work with their hands flexed or twisted and perform repeated finger and arm movements for more than half of the work time (table 1). Both physical and psychosocial factors-including prolonged static muscle activity, insufficient variation in movements and a high degree of precision-are risk factors for development of musculoskeletal disorders. ${ }^{23}{ }^{24}$ Repetitive movements of the arm or wrist are specifically associated with development of forearm pain. ${ }^{4}$ Comparable to the definitions of Kaergaard and coworkers, ${ }^{20}$ we defined pain and disability cases as those scoring more than 30 on a scale of $0-100$. According to this case definition, the prevalence of forearm pain and work disability among the volunteers of the present study was $10 \%$ and $21 \%$, respectively. However, those who agreed to participate in the present study generally had more pain symptoms than those who declined. ${ }^{16}$ Thus, the present study population may not be representative of industrial technicians in general. Kryger and coworkers ${ }^{7}$ reported-based on clinical examination-a prevalence of $4 \%$ of moderate-to-severe forearm pain among computer workers. Although methodological differences exist between our study and Kryger and coworkers, the prevalence of forearm pain may be higher among laboratory technicians than among computer workers in their study. In this regard, Bjorksten and coworkers ${ }^{25}$ showed a higher prevalence of shoulder/hand disorders among laboratory technicians compared with other state employees.

The intention-to-treat analysis, including all participants, showed statistically significant effects of specific resistance training on forearm pain and work disability. As our study included many employees with very low levels of pain and disability, the average changes were small $(4-5$ on a scale of $0-100)$. The rationale for including participants both with and without pain was that musculoskeletal pain typically is episodic. Including all employees allowed us to study the effect of the intervention at the company level, as well as the treatment and preventive effect, respectively, among subgroups with and without pain at baseline. Thus, the results may be important from a public health perspective as it reflects the overall impact of such interventions at a company level.
We also performed subgroup analyses among employees with and without symptoms at baseline to determine a possible treatment and preventive effect, respectively. Among those without symptoms at baseline, there was only minor development of pain and disability in the control group during the 20-week period, which diminishes the statistical power for detecting a possible preventive effect. We have previously shown a preventive effect of a 1-year physical exercise intervention on development of shoulder pain among office workers. ${ }^{21} 26$ Thus, follow-up periods longer than 20 weeks may be necessary to determine a possible preventive effect of resistance training for musculoskeletal pain symptoms. Future studies investigating the preventive effect of physical exercise may consider using follow-up periods of several years. On the other hand, adherence to a specific physical exercise programme is likely to diminish over time.

Among the technicians in the training group with pain $>30 \mathrm{~mm}$ VAS at baseline, pain decreased $20 \mathrm{~mm}$ from baseline to follow-up. An essential part of interpreting these results concerns their clinical relevance. A great heterogeneity between studies exists in the definition of clinically meaningful changes in pain. ${ }^{27}$ However, Farrar and coworkers ${ }^{28}$ found that a change in pain intensity of 1.7 points on a $0-10$ scale discriminates well between patients with pain who on the patient global impression of change scale improved much or very much and patients who did not improve or worsened. Similarly, Dworkin and coworkers ${ }^{29}$ reviewed that a change in pain intensity of 2 points is moderately clinically meaningful and a change of 1 point is the minimally clinically relevant difference. Thus, among those with pain at baseline, the results of our study can be considered clinically relevant. Similar positive results were obtained for work disability. The odds for complete relief of forearm pain and disability were four to sixfolds higher in the training group compared with the control group. Thus, specific resistance training of the upper extremities effectively reduces forearm pain and work disability among employed technicians with symptoms. Randomised controlled trials have shown mixed results on the effectiveness of specific resistance training for specific arm disorders in patients, for example, lateral epicondylitis. ${ }^{12}{ }^{13}$ The study by Svernlöv and Adolfsson ${ }^{13}$ showed that eccentric strengthening exercise performed through a 12-week period was superior to conventional low-intensity stretching exercise. By contrast, Martinez-Silvestrini and coworkers ${ }^{12}$ found that a 6 -week period of strengthening exercise was not more effective than stretching.

In our study, based on the weekly pain registrations, the time-wise change in forearm pain occurred in a roughly linear fashion and became statistically significant after 10 weeks. Likewise, Coury and coworkers ${ }^{11}$ reviewed that physical exercise interventions for musculoskeletal disorders lasting $<10$ weeks rarely find significant results. Thus, workplace interventions with physical 
exercise should last at least 10 weeks to effectively reduce musculoskeletal pain symptoms. Additionally, our results indicate a possible preventive effect for participants without pain at baseline since those in the training group did neither increase pain nor disability while such changes occurred for the control group. However, in the present study group, differences did not attain statistical significance, which may be due to the limited intervention time period.

The pathophysiology of forearm disorders is multifactorial, and some evidence exists for an occupational association. ${ }^{4-6}$ In this regard, prolonged muscle fibre activation and local hypoxia during repetitive arm/hand work tasks may contribute to development of pain symptoms. ${ }^{30} 31$ Using the needle biopsy technique and histochemistry, we have previously found increased proportion of very large slow twitch fibres with poor capillarisation-type I megafibres-in chronically painful neck muscles. ${ }^{32}$ Whether such findings hold true for painful forearm muscles remain unknown. Boyer and Hastings ${ }^{33}$ reported in a review that surgeons commonly observe degenerative changes of the forearm muscles when treating lateral epicondylitis. Regardless of the underlying pathophysiology of forearm pain, we observed reductions of pain and work disability in response to resistance training. Muscle strength increased as evidenced by a doubling of training loads during the 20-week period. As gains in strength lowers the relative exposure of the muscles during work tasks, ${ }^{34}$ strength gains may have contributed to the observed reduction of pain and work disability. Adaptations of the connective tissue in response to the high forces exerted during resistance training may also add to the observed improvements. ${ }^{35}$ The present strengthening exercises involved both concentric and eccentric muscle contractions, that is, raising and lowering the weight in a controlled manner. Studies have shown promising pain reducing effects along with increased peritendinous type I collagen synthesis in response to eccentric training. ${ }^{36}$ Future studies should investigate the multitude of potential pain reducing mechanisms of different types of resistance training for musculoskeletal disorders.

Our study has both strengths and limitations. The cluster randomised controlled design with high statistical power strengthens our study. As we included both public and private sector companies of which most of the invited employees agreed to participate, the external validity of our findings is high. However, the inclusion and exclusion criteria limit the generalisability to technicians with non-specific forearm pain. Although we did not include a clinical examination for diagnosing specific forearm disorders, our results stress the importance of specific resistance training for reducing the overall level of self-reported pain and work disability at the company level. Another limitation is the possible influence of placebo in behavioural interventions. Because we used subjective rating scales and were not able to blind participants, the results may be biased by placebo effects. ${ }^{37}$ Another limitation is the loss to followup of more than $20 \%$ of the participants in the training group. However, using the GLIMMIX procedure to account for missing values at follow-up strengthens the validity of our estimates.

In conclusion, specific resistance training of the shoulder, neck and arm reduces forearm pain and work disability among industrial technicians.

Contributors All authors contributed to conception/design, acquisition of data or analyses and interpretation of data. LLA drafted the article and all co-authors revised it critically for important intellectual content. All authors approved the final version to be published. Thanks to statistician Ole Olsen for performing the randomisation of clusters.

Funding This work was supported by the Danish Working Environment Research Fund (Grant 8-2007-3). The contribution in terms of manpower allowing employees to train during work time for $1 \mathrm{~h} /$ week for 20 weeks was given by the workplaces involved.

Competing interests ICMJE conflicts of interest form for each author of this manuscript.

Ethics approval The ethics approval was approved by Local ethical committee. Provenance and peer review Not commissioned; externally peer reviewed.

Data sharing statement Exploratory analyses from the study is under preparation by the research group. As such, the data set is not yet ready to be shared.

\section{REFERENCES}

1. Andersen LL, Mortensen OS, Hansen JV, et al. A prospective cohort study on severe pain as a risk factor for long-term sickness absence in blue- and white-collar workers. Occup Environ Med 2011;68:590-2.

2. Punnett L, Wegman DH. Work-related musculoskeletal disorders: the epidemiologic evidence and the debate. J Electromyogr Kinesiol 2004;14:13-23.

3. Baldwin ML. Reducing the costs of work-related musculoskeletal disorders: targeting strategies to chronic disability cases. $J$ Electromyogr Kinesiol 2004;14:33-41.

4. Nahit ES, Taylor S, Hunt IM, et al. Predicting the onset of forearm pain: a prospective study across 12 occupational groups. Arthritis Rheum 2003;49:519-25.

5. Fan ZJ, Silverstein BA, Bao S, et al. Quantitative exposure-response relations between physical workload and prevalence of lateral epicondylitis in a working population. Am J Ind Med 2009;52:479-90.

6. van Rijn RM, Huisstede BM, Koes BW, et al. Associations between work-related factors and specific disorders at the elbow: a systematic literature review. Rheumatology (Oxford) 2009;48:528-36.

7. Kryger Al, Andersen JH, Lassen CF, et al. Does computer use pose an occupational hazard for forearm pain; from the NUDATA study. Occup Environ Med 2003;60:e14.

8. Macfarlane GJ, Hunt IM, Silman AJ. Role of mechanical and psychosocial factors in the onset of forearm pain: prospective population based study. BMJ 2000;321:676-9.

9. Feveile $H$, Jensen $C$, Burr $H$. Risk factors for neck-shoulder and wristhand symptoms in a 5-year follow-up study of 3,990 employees in Denmark. Int Arch Occup Environ Health 2002;75:243-51.

10. Pedersen BK, Saltin B. Evidence for prescribing exercise as therapy in chronic disease. Scand J Med Sci Sports 2006;16(Suppl 1):3-63.

11. Coury HJ, Moreira RF, Dias NB. Evaluation of the effectiveness of workplace exercise in controlling neck, shoulder and low back pain: a systematic review. Rev Bras Fisioter 2009;13:461-79.

12. Martinez-Silvestrini JA, Newcomer KL, Gay RE, et al. Chronic lateral epicondylitis: comparative effectiveness of a home exercise program including stretching alone versus stretching supplemented with eccentric or concentric strengthening. J Hand Ther 2005;18:411-19, quiz.

13. Svernlöv B, Adolfsson L. Non-operative treatment regime including eccentric training for lateral humeral epicondylalgia. Scand $J$ Med Sci Sports 2001;11:328-34.

14. Andersen LL, Christensen KB, Holtermann A, et al. Effect of physical exercise interventions on musculoskeletal pain in all body regions among office workers: a one-year randomized controlled trial. Man Ther 2010;15:100-4. 
15. Campbell MK, Elbourne DR, Altman DG; CONSORT group. CONSORT statement: extension to cluster randomised trials. BMJ 2004;328:702-8.

16. Zebis MK, Andersen LL, Pedersen MT, et al. Implementation of neck/ shoulder exercises for pain relief among industrial workers: a randomized controlled trial. BMC Musculoskelet Disord 2011;12:205

17. Huskisson EC. Measurement of pain. Lancet 1974;2:1127-31.

18. Kitis A, Celik E, Aslan UB, et al. DASH questionnaire for the analysis of musculoskeletal symptoms in industry workers: a validity and reliability study. Appl Ergon 2009;40:251-5.

19. Beaton DE, Katz JN, Fossel AH, et al. Measuring the whole or the parts? validity, reliability, and responsiveness of the disabilities of the arm, shoulder and hand outcome measure in different regions of the upper extremity. J Hand Ther 2001;14:128-46.

20. Kaergaard $\mathrm{A}$, Andersen $\mathrm{JH}$, Rasmussen $\mathrm{K}$, et al. Identification of neck-shoulder disorders in a 1 year follow-up study. Validation of a questionnaire-based method. Pain 2000;86:305-10.

21. Blangsted AK, Søgaard K, Hansen EA, et al. One-year randomized controlled trial with different physical-activity programs to reduce musculoskeletal symptoms in the neck and shoulders among office workers. Scand J Work Environ Health 2008;34:55-65.

22. Moher D, Hopewell S, Schulz KF, et al. CONSORT 2010 explanation and elaboration: updated guidelines for reporting parallel group randomised trials. BMJ 2010;340:c869.

23. Sluiter JK, Rest KM, Frings-Dresen MH. Criteria document for evaluating the work-relatedness of upper-extremity musculoskeletal disorders. Scand J Work Environ Health 2001;27 (Suppl 1):1-102.

24. Kilbom A. Repetitive work of the upper extremity: Part I - Guidelines for the practitioner. Int $J$ Ind Erg 1994;14:51-7.

25. Björksten MG, Almby B, Jansson ES. Hand and shoulder ailments among laboratory technicians using modern plunger-operated pipettes. Appl Ergon 1994;25:88-94.
26. Andersen LL, Jorgensen MB, Blangsted AK, et al. A randomized controlled intervention trial to relieve and prevent neck/shoulder pain. Med Sci Sports Exerc 2008;40:983-90.

27. Ruyssen-Witrand A, Tubach F, Ravaud P. Systematic review reveals heterogeneity in definition of a clinically relevant difference in pain. $J$ Clin Epidemiol 2011:64:463-70.

28. Farrar JT, Young JP Jr, LaMoreaux L, et al. Clinical importance of changes in chronic pain intensity measured on an 11-point numerical pain rating scale. Pain 2001:94:149-58.

29. Dworkin RH, Turk DC, McDermott MP, et al. Interpreting the clinical importance of group differences in chronic pain clinical trials: IMMPACT recommendations. Pain 2009;146:238-44.

30. Søgaard K, Sjøgaard G, Finsen L, et al. Motor unit activity during stereotyped finger tasks and computer mouse work. J Electromyogr Kinesiol 2001;11:197-206.

31. Larsson B, Søgaard K, Rosendal L. Work related neck-shoulder pain a review on magnitude, risk factors, biochemical characteristics, clinical picture and preventive interventions. Best Pract Res Clin Rheumatol 2007;21:447-63.

32. Andersen LL, Suetta C, Andersen JL, et al. Increased proportion of megafibers in chronically painful muscles. Pain 2008;139:588-93.

33. Boyer MI, Hastings $\mathrm{H}$. Lateral tennis elbow: "Is there any science out there?". J Shoulder Elbow Surg 1999:8:481-91.

34. Andersen LL, Andersen $\mathrm{CH}$, Zebis MK, et al. Effect of physical training on function of chronically painful muscles: a randomized controlled trial. J Appl Physiol 2008;105:1796-801.

35. Kjaer M, Langberg $\mathrm{H}$, Heinemeier $\mathrm{K}$, et al. From mechanical loading to collagen synthesis, structural changes and function in human tendon. Scand J Med Sci Sports 2009;19:500-10.

36. Langberg $\mathrm{H}$, Ellingsgaard $\mathrm{H}$, Madsen $\mathrm{T}$, et al. Eccentric rehabilitation exercise increases peritendinous type I collagen synthesis in humans with Achilles tendinosis. Scand J Med Sci Sports 2007;17:61-6.

37. Hróbjartsson A, Gøtzsche PC. Placebo interventions for all clinical conditions. Cochrane Database Syst Rev 2004;(3):CD003974. 